\title{
ON THE COFINALITY OF ULTRAPOWERS
}

\author{
AndREAS Blass \\ AND \\ HeIKe MiLdENBERGER
}

\begin{abstract}
We prove some restrictions on the possible cofinalities of ultrapowers of the natural numbers with respect to ultrafilters on the natural numbers. The restrictions involve two cardinal characteristics of the continuum, the splitting number $\mathfrak{s}$ and the groupwise density number $\mathfrak{g}$.
\end{abstract}

\section{INTRODUCTION}

All ultrafilters considered in this paper are non-principal ultrafilters on the set $\omega$ of natural numbers. We shall be concerned with the possible cofinalities $\operatorname{cf}(\mathcal{U}$-prod $\omega)$ of ultrapowers of $\omega$ with respect to such ultrafilters. We shall show that no cardinal below the groupwise density number $\mathfrak{g}$ (see definition below) can occur as such a cofinality and that at most one cardinal below the splitting number $\mathfrak{s}$ can so occur. The proof for $\mathfrak{s}$, when combined with a result of Nyikos, gives the additional information that all $P_{\mathfrak{b}^{+}}$-point ultrafilters are nearly coherent.

In Section 2, we review the necessary terminology and some previously known results. In Section 3, we prove the result concerning $\mathfrak{g}$. Finally, in Section 4, we prove the result concerning $\mathfrak{s}$, we show that in the statement of that result "at most one cardinal" cannot be improved to "no cardinal," and we deduce the result about $P_{\mathfrak{b}^{+}-\text {points. }}$

We thank Simon Thomas for posing the question whether $\operatorname{cf}(\mathcal{U}$-prod $\omega)$ can ever be smaller than $\mathfrak{g}$.

\section{Preliminaries}

We write $\exists^{\infty}$ and $\forall^{\infty}$ for the quantifiers "there exist infinitely many" and "for all but finitely many," respectively. Any ultrafilter (by which we always mean a nonprincipal ultrafilter on $\omega) \mathcal{U}$ will also be used as a quantifier meaning "for almost all with respect to $\mathcal{U}$," i.e.,

$$
(\mathcal{U} n) \varphi(n) \Longleftrightarrow\{n \mid \varphi(n)\} \in \mathcal{U} \text {. }
$$

Thus, the quantifier $\mathcal{U}$ is intermediate between $\forall^{\infty}$ and $\exists^{\infty}$ in the sense that $\left(\forall^{\infty} n\right) \varphi(n) \Longrightarrow(\mathcal{U} n) \varphi(n) \Longrightarrow\left(\exists^{\infty} n\right) \varphi(n)$ for any predicate $\varphi$ on natural numbers.

1991 Mathematics Subject Classification. 03E05.

The first author is partially supported by National Science Foundation grant DMS-9505118 and the second by a postdoctoral fellowship from the Deutsche Forschungsgemeinschaft. 
The ultrapower $\mathcal{U}$-prod $\omega$ is formed from the set ${ }^{\omega} \omega$ of all functions $f: \omega \rightarrow \omega$ by identifying $f$ with $g$ whenever $(\mathcal{U} n) f(n)=g(n)$. It is linearly ordered by the relation

$$
f \leq_{\mathcal{U}} g \Longleftrightarrow(\mathcal{U} n) f(n) \leq g(n) .
$$

By $\operatorname{cf}(\mathcal{U}$-prod $\omega)$ we mean the cofinality of this ordering, the smallest cardinality of a subset $\mathcal{C}$ of ${ }^{\omega} \omega$ such that every $f \in{ }^{\omega} \omega$ is $\leq_{\mathcal{U}}$ some $g \in \mathcal{C}$.

This cofinality obviously satisfies $\mathfrak{b} \leq \operatorname{cf}(\mathcal{U}$-prod $\omega) \leq \mathfrak{d}$, where the bounding number $\mathfrak{b}$ and the dominating number $\mathfrak{d}$ are defined as follows. (For more information on these and other cardinal characteristics of the continuum, see the survey papers $[7,11]$.) $\mathfrak{d}$ is the minimum size of a family $\mathcal{D} \subseteq{ }^{\omega} \omega$ such that, for each $f \in{ }^{\omega} \omega$ there is some $g \in \mathcal{D}$ satisfying $\left(\forall^{\infty} n\right) f(n) \leq g(n)$. The definition of $\mathfrak{b}$ is the same except that $\forall^{\infty}$ is replaced with $\exists^{\infty}$.

In addition to $\mathfrak{b}$ and $\mathfrak{d}$, three other cardinal characteristics of the continuum, $\mathfrak{s}$, $\mathfrak{g}$, and $\operatorname{cov}(\mathrm{B})$, will play a role in this paper.

The splitting number $\mathfrak{s}$ is defined as the minimum size of a family $\mathcal{S}$ of subsets of $\omega$ such that every infinite $X \subseteq \omega$ is split by some $Y \in \mathcal{S}$ in the sense that both $X \cap Y$ and $X-Y$ are infinite.

To define $\mathfrak{g}$, we first need the notion of groupwise density. A family $\mathcal{G}$ of infinite subsets of $\omega$ is said to be groupwise dense if it is closed under infinite subsets and finite modifications and if, whenever $\omega$ is partitioned into finite intervals, the union of some infinitely many of these intervals is in $\mathcal{G}$. Then $\mathfrak{g}$ is defined as the minimum number of groupwise dense families with empty intersection. (See [3] for more information about groupwise density and g.)

Finally, $\operatorname{cov}(\mathrm{B})$ is defined to be the minimum number of meager sets (i.e., sets of the first Baire category) needed to cover the real line.

We shall be concerned with restrictions, in terms of cardinal characteristics of the continuum, on the possible values of $\mathrm{cf}(\mathcal{U}$-prod $\omega)$. The following theorem of Canjar $[4,5]$ and Roitman [9] suggests that the trivial restriction $\mathfrak{b} \leq \operatorname{cf}(\mathcal{U}$-prod $\omega) \leq \mathfrak{d}$ is all one can hope for.

Theorem $1[4,5,9]$. It is consistent (relative to $Z F C$ ) that $\mathfrak{b} \ll \mathfrak{d}$ and every regular cardinal $\kappa$ in the range $\mathfrak{b} \leq \kappa \leq \mathfrak{d}$ occurs as $\operatorname{cf}(\mathcal{U}$-prod $\omega)$ for some $\mathcal{U}$.

The model used to prove this theorem is the Cohen model, obtained by adding a large number of Cohen-generic reals to any model of ZFC. We shall see that the trivial lower bound $\mathfrak{b}$ for all $\operatorname{cf}(\mathcal{U}$-prod $\omega)$ can be improved in some models (but not in all, by Theorem 1).

Canjar also showed that the trivial upper bound $\mathfrak{d}$ cannot be improved in any model where $\mathfrak{d}$ is regular.

Theorem 2 [6]. There exists an ultrafilter $\mathcal{U}$ with $c f(\mathcal{U}$-prod $\omega)=c f(\mathfrak{d})$. In particular, if $\mathfrak{d}$ is regular then it occurs as cf $(\mathcal{U}$-prod $\omega)$ for some $\mathcal{U}$.

For any ultrafilter $\mathcal{U}$ and any function $f: \omega \rightarrow \omega$, the image $f(\mathcal{U})$ is defined as the ultrafilter $\left\{X \subseteq \omega \mid f^{-1}(X) \in \mathcal{U}\right\}$. (Contrary to our convention, this may be a principal ultrafilter, but only if $f$ is constant on some set in $\mathcal{U}$; we shall use $f(\mathcal{U})$ only for finite-to-one functions $f$, so no real difficulty arises.) Two ultrafilters $\mathcal{U}$ and $\mathcal{U}^{\prime}$ are said to be nearly coherent if $f(\mathcal{U})=f^{\prime}\left(\mathcal{U}^{\prime}\right)$ for some finite-to-one functions $f$ and $f^{\prime}$. It is shown in [1] that the same relation of near coherence would be 
also shown there that near coherence is an equivalence relation and that, whenever $\mathcal{U}$ and $\mathcal{U}^{\prime}$ are nearly coherent, then $\operatorname{cf}(\mathcal{U}-\operatorname{prod} \omega)=\operatorname{cf}\left(\mathcal{U}^{\prime}-\operatorname{prod} \omega\right)$ (because both of these ultrapowers have cofinal submodels isomorphic to $f(\mathcal{U})$-prod $\omega)$. The principle of near coherence of filters (NCF), introduced in [1] and proved consistent in [2], asserts that every two non-principal ultrafilters on $\omega$ are nearly coherent.

\section{Groupwise Density Gives a Lower Bound}

In this section, we prove the following answer to a question raised by Simon Thomas (private communication).

Theorem 3. For every non-principal ultrafilter $\mathcal{U}$ on $\omega, c f(\mathcal{U}$-prod $\omega) \geq \mathfrak{g}$.

Proof. Suppose $\mathcal{C} \subseteq{ }^{\omega} \omega$ is cofinal with respect to $\leq_{\mathcal{U}}$. We shall associate to each $f \in \mathcal{C}$ a groupwise dense family $\mathcal{G}_{f}$ in such a way that the intersection of these families is empty. Thus, we shall have $\mathfrak{g} \leq|\mathcal{C}|$, which establishes the theorem.

By increasing them if necessary, we may assume without loss of generality that all the functions $f \in \mathcal{C}$ satisfy $f(n) \geq n$ for all $n$. To define $\mathcal{G}_{f}$, we first define, for each infinite $X \subseteq \omega$, the function $\nu_{X}: \omega \rightarrow \omega$ sending each natural number $n$ to the next larger element of $X$. Then let

$$
\mathcal{G}_{f}=\left\{X \subseteq \omega \mid X \text { is infinite and } f<\mathcal{U} \nu_{X}\right\}
$$

for each $f \in \mathcal{C}$. Since these $f^{\prime}$ 's are cofinal in $\mathcal{U}$-prod $\omega$, the intersection of the corresponding $\mathcal{G}_{f}$ 's must be empty. It is also clear that each $\mathcal{G}_{f}$ is closed under infinite subsets and under finite modifications. So to verify that each $\mathcal{G}_{f}$ is groupwise dense, thus completing the proof, it remains only to check that, if $f$ is fixed and if $\omega$ is partitioned into finite intervals then the union of some infinitely many of these intervals is in $\mathcal{G}_{f}$.

Inductively select intervals $I_{k}$ from the given partition so that the first element of $I_{k+1}$ is greater than $f(x)$ for all $x \in I_{k}$ and all smaller $x$. Let $X$ be the union of the even-numbered intervals, $I_{2 j}$, and $Y$ the union of the odd-numbered ones.

For any natural number $p$ in the interval $\left(\max I_{n-1}, \max I_{n}\right]$, one of $\nu_{X}(p)$ and $\nu_{Y}(p)$ (depending on the parity of $n$ ) will be min $I_{n+1}$, which is greater than $f(p)$. Thus, every natural number $p$, except for the finitely many below max $I_{0}$, is in one of the two sets $\left\{n \in \omega \mid f(n)<\nu_{X}(n)\right\}$ and $\left\{n \in \omega \mid f(n)<\nu_{Y}(n)\right\}$. Therefore, one of these sets is in $\mathcal{U}$, which means that one of $X$ and $Y$ is in $\mathcal{G}_{f}$. Since both $X$ and $Y$ are unions of infinitely many intervals from the given partition, this completes the proof that $\mathcal{G}_{f}$ is groupwise dense and thus completes the proof of the theorem.

It is well-known (see [3]) that $\mathfrak{g} \leq \mathfrak{d}$. The following corollary gives an improvement when $\mathfrak{d}$ is singular.

Corollary 4. $\mathfrak{g} \leq c f(\mathfrak{d})$.

Proof. Combine Theorems 2 and 3.

Encouraged by Theorem 3, one might look for additional cardinal characteristics that give lower bounds on the possible cofinalities of $\mathcal{U}$-prod $\omega$. Such characteristics must be $\leq \mathfrak{d}$ and, to avoid trivialities, $\not{E} \mathfrak{b}$. Inspection of the diagrams of cardinal characteristics in [11] provides just two such characteristics, the splitting number $\mathfrak{s}$ and the covering number for category $\operatorname{cov}(\mathrm{B})$. (If one counts the somewhat artificial 
remark about $\operatorname{cov}(\mathrm{B})$ applies to it as well.) If we add a large number $\kappa$ of Cohen reals to a model of set theory, then the resulting model has cov(B) large but has, by the proof of Theorem 1, ultrafilters with $\mathrm{cf}(\mathcal{U}$-prod $\omega)=\aleph_{1}$. So $\operatorname{cov}(\mathrm{B})$ cannot serve as a lower bound for $\operatorname{cf}(\mathcal{U}$-prod $\omega)$. That leaves $\mathfrak{s}$ as a possibility, which we analyze in the next section.

\section{The Splitting Number}

Unlike $\mathfrak{g}$, the splitting number $\mathfrak{s}$ is not in general a lower bound for $\operatorname{cf}(\mathcal{U}$-prod $\omega)$. The proof involves the notion of (pseudo-) $P_{\kappa}$ point. An ultrafilter $\mathcal{U}$ is called a $P_{\kappa}$ point if, for every family $\mathcal{F} \subseteq \mathcal{U}$ with $|\mathcal{F}|<\kappa$, there is some $A \in \mathcal{U}$ with $A-F$ finite for all $F \in \mathcal{F}$. Pseudo- $P_{\kappa}$ points are defined similarly, except that $A$ is not required to be in $\mathcal{U}$, only to be infinite. We shall need the following results of Nyikos, folklore, and Shelah, respectively. (Although Nyikos's paper [8] is not yet published, Proposition 5 and its proof were in a 1984 letter from Nyikos to the first author.)

Proposition 5 [8]. If $\mathcal{U}$ is a pseudo- $P_{\kappa}$ point and $\kappa>\mathfrak{b}$, then $\operatorname{cf}(\mathcal{U}$-prod $\omega)=\mathfrak{b}$.

Proposition 6. If $\mathcal{U}$ is a pseudo- $P_{\kappa}$ point then $\mathfrak{s} \geq \kappa$.

Proposition 7 [2]. It is consistent relative to $Z F C$ that $\mathfrak{b}=\aleph_{1}$ and there is a $P_{\aleph_{2}}$-point.

Since the first two of these propositions are fairly easy, we give their proofs. For Proposition 7, we refer to Theorem 6.1 of [2], which gives (more than) a model with a $P_{\aleph_{2}}$-point and another ultrafilter generated by $\aleph_{1}$ sets. The latter gives us $\mathfrak{b}=\aleph_{1}$ because, by a theorem of Solomon [10], no ultrafilter can be generated by fewer than $\mathfrak{b}$ sets.

Proof of Proposition 5. Let $\mathcal{U}$ be a pseudo- $P_{\kappa}$ point with $\kappa>\mathfrak{b}$, and let $\mathcal{C} \subseteq{ }^{\omega} \omega$ be a family of cardinality $\mathfrak{b}$ such that for every $f \in{ }^{\omega} \omega$ there is $g \in \mathcal{C}$ with $\left(\exists^{\infty} n\right) f(n) \leq$ $g(n)$. By increasing each $g \in \mathcal{C}$ if necessary, we can assume that $g$ is a monotone non-decreasing function. To complete the proof, we show that $\mathcal{C}$ is cofinal with respect to the linear ordering $\leq_{\mathcal{U}}$ of $\mathcal{U}$-prod $\omega$.

Suppose to the contrary that $h \in \omega^{\omega} \omega$ is such that $g \leq_{\mathcal{U}} h$ for all $g \in \mathcal{C}$. This means that the sets $M_{g}=\{n \in \omega \mid g(n) \leq h(n)\}$ are in $\mathcal{U}$ for all $g \in \mathcal{C}$. Since $|\mathcal{C}|=\mathfrak{b}<\kappa$ and since $\mathcal{U}$ is a pseudo- $P_{\kappa}$ point, there is an infinite set $X \subset \omega$ such that each $X-M_{g}$ is finite. As in the proof of Theorem 3 , let $\nu_{X}(n)$ denote the next member of $X$ after $n$. By our original choice of $\mathcal{C}$, there is $g \in \mathcal{C}$ such that $h\left(\nu_{X}(n)\right)<g(n)$ for infinitely many $n$. For each such $n$ we have, since $g$ is nondecreasing, $h\left(\nu_{X}(n)\right)<g\left(\nu_{X}(n)\right)$ and therefore $\nu_{X}(n) \in X-M_{g}$. But this applies to infinitely many $n$, giving infinitely many $\nu_{X}(n)$, contrary to the fact that $X-M_{g}$ is finite.

Proof of Proposition 6. Let $\mathcal{U}$ be a pseudo- $P_{\kappa}$ point and let $\mathcal{S}$ be a family of fewer than $\kappa$ subsets of $\omega$. We must find an infinite set $X \subseteq \omega$ that is not split by any member of $\mathcal{S}$.

For each $Y \in \mathcal{S}$, let $Y^{\prime}$ be $Y$ or $\omega-Y$, whichever is in $\mathcal{U}$. As $\mathcal{U}$ is a pseudo- $P_{\kappa}$ point, there is an infinite $X$ such that $X-Y^{\prime}$ is finite for all $Y \in \mathcal{S}$. This $X$ is clearly not split by any such $Y$. 
Corollary 8. It is consistent, relative to $Z F C$, that there is a non-principal ultrafilter $\mathcal{U}$ on $\omega$ with $c f(\mathcal{U}$-prod $\omega)<\mathfrak{s}$.

Proof. In the model given by Proposition 7 , let $\mathcal{U}$ be a $P_{\aleph_{2}}$ point. Its existence gives $\mathfrak{s} \geq \aleph_{2}$ by Proposition 6 , and we also have, by Propositions 5 and $7, \operatorname{cf}(\mathcal{U}$-prod $\omega)=$ $\mathfrak{b}=\aleph_{1}$.

Although Corollary 8 shows that it is consistent for the set of cofinalities of ultrapowers of $\omega$ to contain a cardinal below $\mathfrak{s}$, we shall see that this set cannot contain two cardinals below $\mathfrak{s}$. That will be a consequence of the following theorem.

Theorem 9. Suppose $\mathcal{U}$ and $\mathcal{U}^{\prime}$ are non-principal ultrafilters on $\omega$ such that both $c f(\mathcal{U}$-prod $\omega)$ and $c f\left(\mathcal{U}^{\prime}\right.$-prod $\left.\omega\right)$ are smaller than $\mathfrak{s}$. Then $\mathcal{U}$ and $\mathcal{U}^{\prime}$ are nearly coherent.

Proof. Let $\mathcal{U}$ and $\mathcal{U}^{\prime}$ satisfy the hypotheses of the theorem, and suppose these ultrafilters are not nearly coherent. Let $\mathcal{C}$ and $\mathcal{C}^{\prime}$ be subfamilies of ${ }^{\omega} \omega$, each of size $<\mathfrak{s}$, and cofinal with respect to $\leq_{\mathcal{U}}$ and $\leq_{\mathcal{U}^{\prime}}$ respectively. Let $\mathcal{D}$ be the set of functions of the form $\max \left\{g, g^{\prime}\right\}$, where $g \in \mathcal{C}, g^{\prime} \in \mathcal{C}^{\prime}$, and max means the pointwise maximum of the functions. Then, for each $f \in{ }^{\omega} \omega$, there is an $h \in \mathcal{D}$ such that both inequalities $f \leq_{\mathcal{U}} h$ and $f \leq_{\mathcal{U}^{\prime}} h$ hold.

Temporarily fix some $h \in \mathcal{D}$. Partition $\omega$ into finite intervals $I_{n}=\left[a_{n}, a_{n+1}\right)$ such that $h(x)<a_{n+1}$ for all $x<a_{n}$. (It is trivial to produce such $a_{0}=0<a_{1}<$ $a_{2}<\ldots$ inductively.) Let $p: \omega \rightarrow \omega$ be the function that sends all points in $I_{n}$ to $n$, for all $n$. Since $p$ is finite-to-one and since $\mathcal{U}$ and $\mathcal{U}^{\prime}$ are not nearly coherent, the ultrafilters $p(\mathcal{U})$ and $p\left(\mathcal{U}^{\prime}\right)$ are distinct, so one contains a set whose complement is in the other. Pulling these sets back along $p$, we get two sets, say $A \in \mathcal{U}$ and $A^{\prime} \in \mathcal{U}^{\prime}$, each a union of some $I_{n}$ 's, but with no $I_{n}$ in common.

Define $q(x)=p(x)+1$. Applying again the fact that $\mathcal{U}$ and $\mathcal{U}^{\prime}$ are not nearly coherent, we have $q(\mathcal{U}) \neq p\left(\mathcal{U}^{\prime}\right)$, so we can get a set in $q(\mathcal{U})$ whose complement is in $p\left(\mathcal{U}^{\prime}\right)$. Pulling these sets back along $q$ and $p$ respectively, we get $B \in \mathcal{U}$ and $B^{\prime} \in \mathcal{U}^{\prime}$, each a union of some $I_{n}$ 's, and such that we never have an $I_{n} \subseteq B$ and $I_{n+1} \subseteq B^{\prime}$.

Arguing analogously with $p(\mathcal{U}) \neq q\left(\mathcal{U}^{\prime}\right)$, we get $C \in \mathcal{U}$ and $C^{\prime} \in \mathcal{U}^{\prime}$, each a union of some $I_{n}$ 's, such that we never have an $I_{n} \subseteq C^{\prime}$ and $I_{n+1} \subseteq C$.

Let $D=A \cap B \cap C$ and $D^{\prime}=A^{\prime} \cap B^{\prime} \cap C^{\prime}$. Then $D \in \mathcal{U}, D^{\prime} \in \mathcal{U}^{\prime}$, and both are unions of some $I_{n}$ 's. Furthermore, if a particular $I_{n}$ is included in $D$ then neither it nor its neighbors $I_{n \pm 1}$ can be included in $D^{\prime}$.

Let $E$ be the union of all the $I_{n}$ 's and $I_{n+1}$ 's such that $I_{n} \subseteq D$, i.e., the union of the intervals that constitute $D$ and their right neighbor intervals. Define $E^{\prime}$ similarly from $D^{\prime}$, and note that $E$ and $E^{\prime}$ are disjoint.

I claim that, if $X$ is an infinite subset of $\omega$ and if $\nu_{X} \leq \mathcal{U} h$, then $X \cap E$ is infinite. To see this, notice first that the set $\left\{k \in \omega \mid \nu_{X}(k) \leq h(k)\right\}$, being in $\mathcal{U}$, must contain infinitely many points $k \in D$ because $D \in \mathcal{U}$. For each of these infinitely many $k$, there is an element of $X$, namely $\nu_{X}(k)$, in the interval $[k, h(k)]$. By our choice of the intervals $I_{n}$, this element of $X$ is either in the same interval as $k$ or in its right neighbor. In either case, it is in $E$ because $k \in D$. Thus, we have infinitely many (since $k$ can be arbitrarily large) elements of $X \cap E$, as claimed.

Similarly, if $\nu_{X} \leq_{\mathcal{U}^{\prime}} h$, then $X \cap E^{\prime}$ is infinite and therefore so is $X-E$ since $E$ 
Now un-fix $h$. For each $h \in \mathcal{D}$, the preceding discussion produces an $E$, which we now call $E_{h}$ to indicate its dependence on the (previously fixed) $h$. For any infinite subset $X$ of $\omega$, the function $\nu_{X}$ is majorized, with respect to both $\leq_{\mathcal{U}}$ and $\leq_{\mathcal{U}^{\prime}}$, by some $h \in \mathcal{D}$. then the preceding discussion shows that $X$ is split by the corresponding $E_{h}$. Therefore, $\left\{E_{h} \mid h \in \mathcal{D}\right\}$ is a splitting family. But this is absurd, as $|\mathcal{D}|<\mathfrak{s}$.

Corollary 10. At most one cardinal smaller than $\mathfrak{s}$ can occur as $\operatorname{cf}(\mathcal{U}$-prod $\omega)$.

Proof. Combine Theorem 9 and the fact that nearly coherent ultrafilters produce ultrapowers of the same cofinality.

Corollary 11. Any two pseudo- $P_{\mathfrak{b}}+$ points are nearly coherent.

Proof. If two ultrafilters are pseudo- $P_{\mathfrak{b}}+$ points, then the corresponding ultrapowers have cofinality $\mathfrak{b}$ by Proposition 5 , and this is smaller than $\mathfrak{s}$ by Proposition 6 . So Theorem 9 applies and gives the required near coherence.

Remark. For an ultrafilter $\mathcal{U}$ to have a small system of generators and for its ultrapower $\mathcal{U}$-prod $\omega$ to have small cofinality are in some sense antithetical properties. Specifically, the proof of Theorem 16 in [1] shows that the number of generators of $\mathcal{U}$ and $\operatorname{cf}(\mathcal{U}$-prod $\omega)$ cannot both be smaller than $\mathfrak{d}$. Yet each property, when it holds of two ultrafilters (with an appropriate sense of "small") implies near coherence. For $\mathrm{cf}(\mathcal{U}$-prod $\omega)$, the appropriate sense of "small" is $<\mathfrak{s}$ and the relevant result is Theorem 9 above. For the number of generators of $\mathcal{U}$, the appropriate sense of "small" is $<\mathfrak{d}$, for Corollary 13 of [1] says that any two ultrafilters generated by fewer than $\mathfrak{d}$ sets are nearly coherent.

\section{REFERENCES}

1. A. Blass, Near Coherence of filters, I: Cofinal equivalence of models of arithmetic, Notre Dame J. Formal Logic 27 (1986), 579-591.

2. A. Blass and S. Shelah, There may be simple $P_{\aleph_{1}}$ - and $P_{\aleph_{2}}$-points and the Rudin-Keisler ordering may be downward directed, Ann. Pure Appl. Logic 33 (1987), 213-243.

3. A. Blass, Applications of superperfect forcing and its relatives, Set Theory and its Applications (J. Steprāns and S. Watson, eds.), Lecture Notes in Mathematics 1401, Springer-Verlag, 1989, pp. 18-40.

4. R. M. Canjar, Model-Theoretic Properties of Countable Ultraproducts Without the Continuum Hypothesis, Ph.D. Thesis, University of Michigan, 1982.

5. R. M. Canjar, Countable ultraproducts without CH, Ann. Pure Appl. Logic 37 (1988), 1-79.

6. R. M. Canjar, Cofinalities of countable ultraproducts: the existence theorem, Notre Dame J. Formal Logic 30 (1989), 539-542.

7. E. van Douwen, The integers and topology, Handbook of Set Theoretic Topology (K. Kunen and J. Vaughan, eds.), North-Holland, 1984, pp. 111-167.

8. P. Nyikos, Special ultrafilters and cofinal subsets of ${ }^{\omega} \omega$ (to appear).

9. J. Roitman, Non-isomorphic H-fields from non-isomorphic ultrapowers, Math. Z. 181 (1982), 93-96.

10. R. C. Solomon, Families of sets and functions, Czechoslovak Math. J. 27 (1977), 556-559.

11. J. Vaughan, Small uncountable cardinals and topology, Open Problems in Topology (J. van Mill and G. Reed, eds.), North-Holland, 1990, pp. 195-218.

Mathematics Dept., University of Michigan, Ann Arbor, Mi 48109, U.S.A.

E-mail address: ablass@umich.edu, mildenbe@umich.edu 